\title{
L'appareil urinaire du chameau
}

\author{
par TAYEB (M.A.F.) \\ Professeur d'Anatomie, Collège de médecine vétérinaire de Giza (Égypte) (1)
}

\section{TRAVAUX ANTÉRIEURS}

Chauveau indique que les reins du chameau ne sont pas lobulés et que leur bassinet rappelle celui du rein du cheval, faisant transition toutefois entre le bassinet du rein des Solipèdes et le bassinet du rein de bouf.

LESBRE précise que les reins du chameau ont la forme du rein des ovins ou des caprins; ils sont volumineux; le rein gauche pesant 800 grammes et le droit 750 grammes. Le rein droit est en position antérieure par rapport au rein gauche et le bord postérieur du premier se trouve au même niveau que le bord antérieur du second. Le rein gauche est refoulé vers l'entrée du bassin par le rumen et le rein droit s'appuie contre le bord supérieur du foie. La capsule fibreuse est épaisse, opaqué, adhérente au tissu sous-jacent; la couche médullaire forme une crête unipapillaire comme chez le mouton, la chèvre, le chien et le cheval.

Les irradiations de la muqueuse pyélique constituent un labyrinthe complexe.

L'artère rénale gauche est oblique et envoie une branche collatérale à la rate.

L'uretère du chameau rappelle celui des autres especes et la vessie offre une paroi peu épaisse.

LEESE, enfin, établit que les reins du chameau sont gros, non lobulés, de forme et de dimensions semblables à gauche et à droite.

Le rein droit est en position antérieure.

Les deux reins pesent 900 grammes ( $2 \mathrm{lb}$ ) chacun. La vessie est de faible capacité.

\section{MATÉRIEL D'ÉTUDE}

Les organes décrits ont été prélevés aux abattoirs $\mathrm{du}$ Caire. I'appareil urinaire a été étudié sur 50 chameaux soudanais adultes, mâles et femelles; les organes ont été décrits, pesés et mesurés à l'état frais, puis mis en solution formolée à $10 \%$ en vue d'un examen ultérieur.

Quelques reins furent injectés par l'artère rénale

(1) Traduction par P.-C. Blin, Assistant d'Anatomie, Alfort. avec une solution formolée colorée afin d'établir le circuit vasculaire de ces organes.

L'étude in situ permit de préciser leur position et leurs rapports.

2 chameaux furent saignés puis injectés par l'artère carotide avec la masse à injection suivante :

\begin{tabular}{|c|c|c|}
\hline acide arsénieux & & 200 grammes \\
\hline carbonate de sodiu & $\mathrm{am}$. & 200 grammes \\
\hline acide phénique pu & & $2 \mathrm{kgr} .500$ \\
\hline glycérine & . & $2 \mathrm{kgr} .500$ \\
\hline formol & $\ldots \ldots$ & $2 \mathrm{kgr} .500$ \\
\hline eau chaude & $\ldots \ldots \ldots$ & 25 litres \\
\hline
\end{tabular}

On mit du bleu de Prusse en quantité suffisante pour colorer le mélange et, lors de l'injection, on ajouta 2 kilogrammes de plâtre, progressivement, en remuant fortement, en vue de la mise en évidence des artères.

On fit également l'examen microscopique des organes urinaires.

\section{LES REINS}

Les reins se présentent lisses, non lobulés, en forme de haricot. Ils ont une face dorsale plane et une face ventrale convexe.

Le bord externe est arrondi à la fois horizontalement et dorso-ventralement.

Le bord interne est arrondi et profondément échancré, non pas exactement en son milieu, mais au niveau du hile, de sorte qu'une coupe segmentale passanl à ce niveau divise le rein en deux parties inégales.

La partie antérieure du rein droit est plus grande que celle du rein gauche.

Le pôle antérieur du rein gauche est acuminé, le pôle postérieur, au contraire, est arrondi, de sorte que le contour de l'organe rappelle celui d'une oreille humaine.

Par suite de sa longueur plus faible, de sa sphéricité plus accusée, le rein droit apparait de forme globuleuse. La portion ventrale de l'artère rénale gauche se divise en deux branches au contact même du hile du rein gauche.

Sur tous les sujets étudiés, le rein gauche était plus volumineux que le rein droit. 


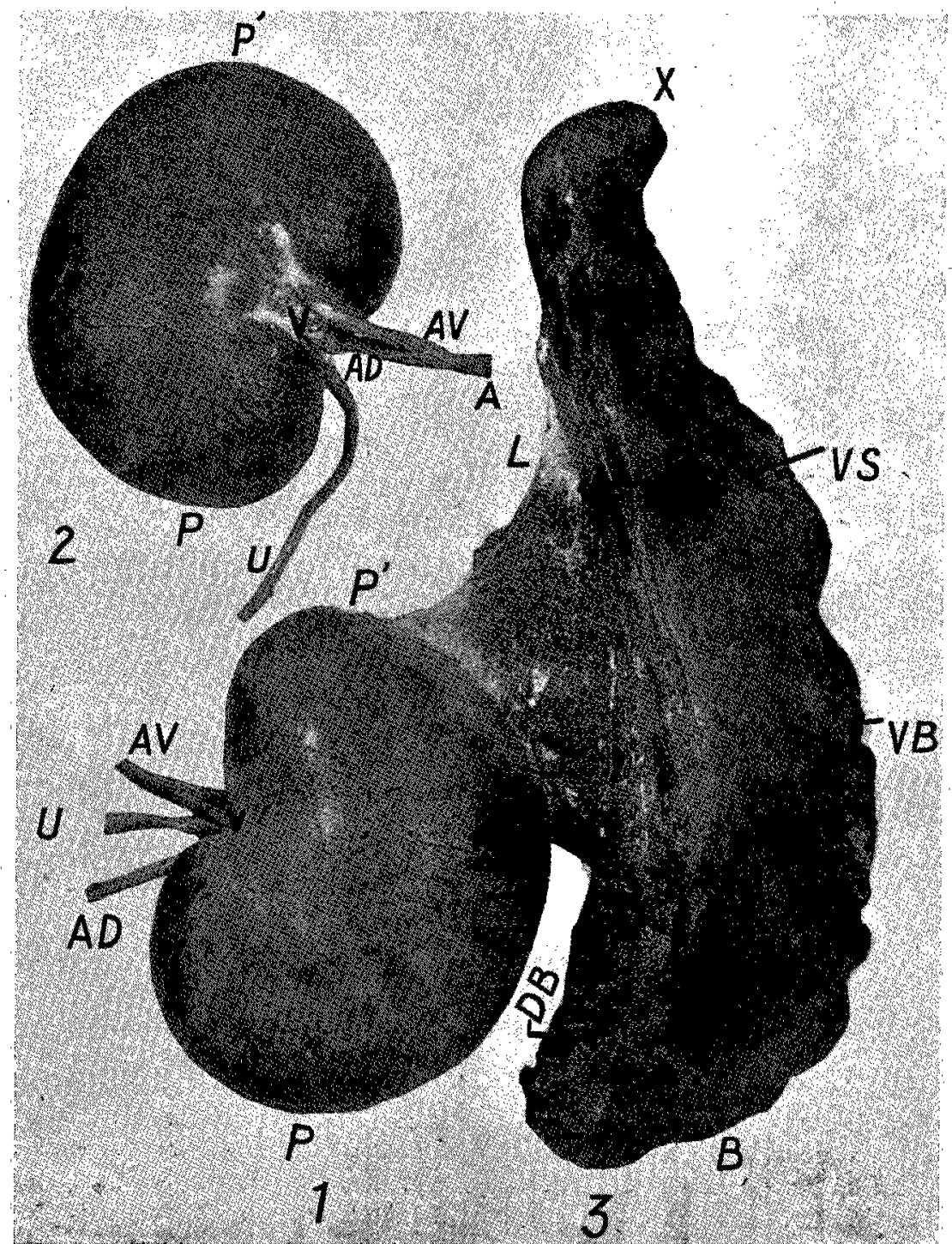

$$
\text { Fig. . - Reins }
$$

1. Rein gauche. z. Rein droit. 3. Rate

1. Rein gauche.

'P'; póle antérieur. - V, veine rénale. - AV, branche ventrale de l'artèrẹ rénale. U, uretère. - AD, branche dorsale de l'artère répale. - P, póle postérieur.

R. Rein droit.

'P', póle antérieur. - $\boldsymbol{A}$, artère rénale droite. - $\mathbf{A V}$, sa branche ventrale. $-\mathbf{A D}$, sa branche dorsale. - U, uretère. - P, póle postérieur.

3. Rate.

$\mathbf{X}$ apex de la rate. - Vs, face interne, - VB, bord inférieur. - DB, bord supérieur. L. ligament splénique. 


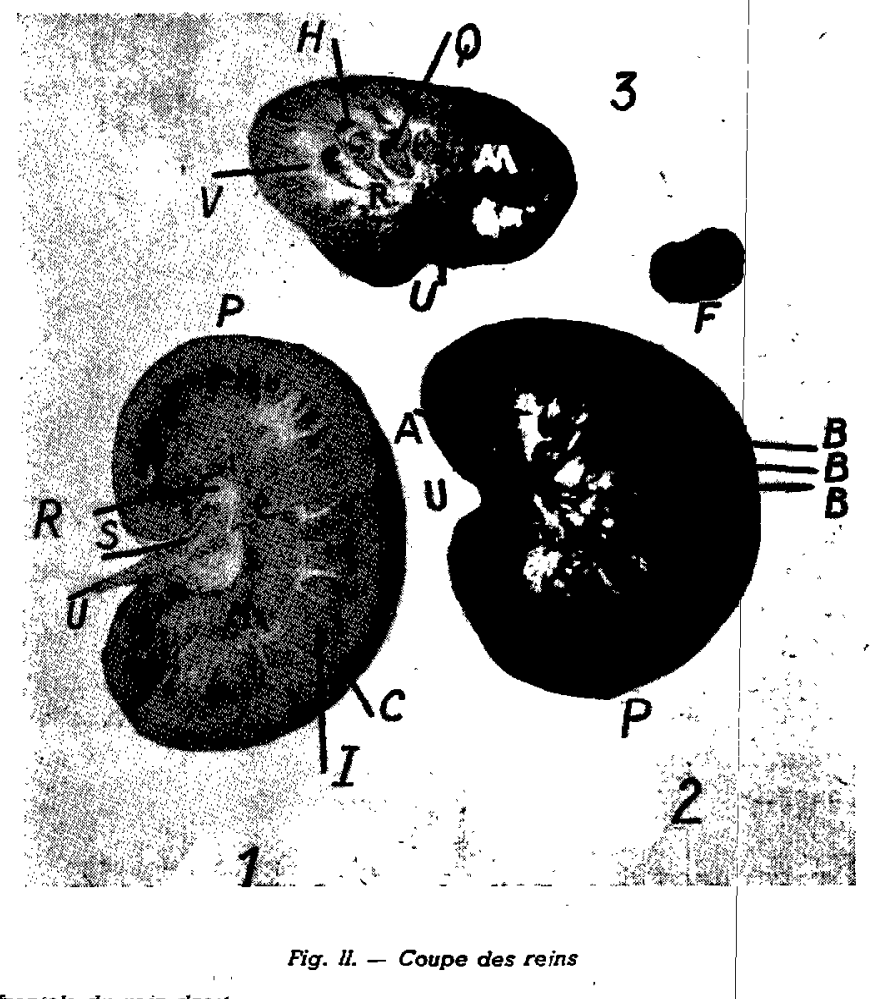

1. Coupe frontale du rein drout.

$\mathbf{R}$ bassine $-\mathbf{S}$, sinus rénal. "- $\mathbf{e}$, sonde engagée dans l'uretère et reposant sur la créte pyélique (U) - M, substance médullaire. - C. cortex. - I, zone intermédiaire - P, póle antérieur

2. Coupe frontale du rein gauche (à un niveau plus élevé que dans la fig. 1).

$\mathbf{B}$ arcades rénale se rattachant au cortex - $\mathbf{-}$, une des branches de l'artère rénale envoyant ses divisions entre les irradiations des arcades rénales. -. $\mathbf{0}$. uretère. - $\mathbf{P}$, póle postérieur.

3. Coupe frontale et segmentale d'un rein.

$\mathbf{T}$, arcades verticales - $\mathbf{H}$ arcades horizontales. - Q. locuti. - $\mathbf{M}$, substance médullaire (en partie reséquée) - $\mathbf{R}$, bassinet. - $\mathbf{U}$, uretère. - $\mathbf{F}$, reins de foetus (de $50 \mathrm{~cm}$.).

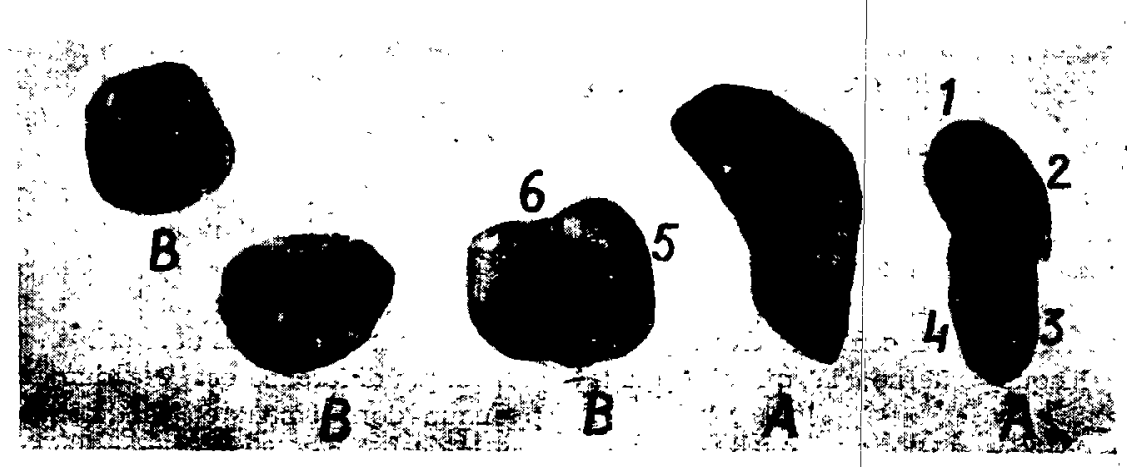

Fig II. - Glandes surrenales

A. Surrénale droite. B Surténale gauche

Surtenale drote. - 1. Pole anterieur. 2. Partie antérieure du bord interne. 3. Partie postérieure du bord interne. 4 Bord externe.

Surrénale gauche. - 5. Partie antérieure. 6. Bord in'erne. 
Chez les adultes des deux sexes, le poids moyen du rein gauche est de 1000 grammes et les dimensions environ $19 \times 12 \times 9$ centimètres. Le rein droit pèse environ 900 grammes et mesure $18 \times 10,5$ $\times 11 \times 7,8$ centimètres.

Des reins examinés, le plus volumineux, un rein gauche, accusait un poids de 1.462 gr. 5 et mesurait $22 \times 12,5 \times 8$ centimètres. Le rein droit le plus volumineux accusait un poids de 1.350 grammes et mesurait $20,5 \times 10 \times 8$ centimètres.

Rein droit et rein gauche sont donc dissemblables, contrairement à ce que disent Lesbre et Leese.

Les deux reins'sont noyés dans une capsule adipeuse dont le développement dépend de l'état de l'animal. A la différence des autres espèces sur un reị normal à l'état frais,' la capsule fibreuse rénale (tunica fibrosa) est si dense et si opaque que le rein intact apparaît, grisâtre ou blanc bleuté. Elle est très adhérente au cortcx rénal sous-jacent et quand on la détache, ce dernier perd son caractère lisse et présente des flammèches à sa surface.

\section{POSITION ET RAPPORTS}

Le rein droit s'étend sous les premier, deuxième et troisième espaces lombaires. Il est en rapport, en haut, avec le petit et le grand psoas; en bas, avec le duodénum et la portion supérieure du côlon. Le bord interne est en contact avec la veine cave, sauf sa partie antéro-supérieure qui est coiffée par la surrénale droite.

Le bord externe est en rapport avec la paroi abdominale.

«L'impression rénale » au niveau du foie est si marquée, qu'on peut la qualifier de fosse rénale chez le chameau.

Le rein gauche est situé sous les apophyses transverses des cinquième, sixième et septième vertèbres lombaires. En haut, il est en rapport avec les muscles psoas.

En arrière et latéralement, la face inférieure est en rapport avec le côlon spirale et, en avant, avec la face supérieure du rumen. Le côté supérieur de la rate se moule sur le bord externe du rein gauche. Le bord interne de cet organe entre en contact avec l'aorte, sauf en arrière où il contracte des rapports avec l'artère iliaque externe, là où elle quitte l'aorte.

Le bord postérieur touche l'inflexion du côlon sigmoide.

Contrairement à ce qu'on lit dans Lesbre, le bord antérieur du rein gauche n'est pas au même niveau que le bord postérieur du rein droit.

A la différence de ce qu'on voit chez le boeuf, le rein gauche du chameau occupe une position supérieure et est beaucoup moins flottant, par suite du faible développement du fascia rénal; de sorte que, ' dans l'indigestion par surcharge du rumen, l'organe n'est pas déplacé, ni vers la droite, ni en arrière, vers l'entrée du bassin.

Par suite de sa position postérieure, le rein gauche peut être exploré par voie rectale.

\section{LES ARTÈRES RÉNALES}

L'artère rénale droite nât sùr l'áorle latéralement, en ligne par rapport à l'artère grande mésentérique, mais un peu plus haut, sous la première vertèbre lombaire.

Elle passe à droite, au-dessus de la veine cave et atteint le rein droit.

Au niveau même de la face externe de la veine cave, l'artère se divise en deux branches : une dorsale et une ventrale. La première pénètre le hile, dorsalement par rapport à l'uretère, la deuxième ventralement.

L'artère rénale gauche prend son origine à partir de la face externe de l'aorte, au voisinage de sa face inférieure, postérieurement par rapport à l'artère grande mésentérique, sous la deuxième vertèbre lombaire. Elle a un trajet oblique d'avant en arrière et légèrement de haut én bas; sa longueur est de 16-17 centimètres. Après 2 ou 3 centimètres, elle se divise en deux branches comme l'artere rénale droite.

Elle ne présente pas de branche collatérale pour. la rate. Les veines rénales sont situées ventralement au niveau du hile.

\section{STRUCTURE DU REIN}

Des coupes de rein frais offront la même structurc d'ensemble que dans les autres espèces; le cortex rénal est brun foncé; la zone intermédiaire est d'aspect brillant, rouge foncé; la substance médullaire est brun clair.

Le sinus rénal forme un espace triangulaire dont la pointe se trouve au niveau du hile.

Le bassinet est une cavité étroite, en croissant, et la zone médullaire forme une crête saillante comme chez le mouton, le chien et le cheval.

L'unique différence est l'étendue de la muqueuse pyélique dorsalement et ventralement, qui forme environ douze irradiations divergeant vers la grande courbure de l'organe (Photo II). Chaque irradiation est plissée et comprend deux couches qui enserrent les vaisseaux sanguins.

Les irradiations ventrales et dorsales 's'unissent latéralement et forment des arcades dont le bord 
convexe se rattache à la substance corticale, tandis que le bord concave, arrondi, regarde la substance médúllaire.

Les arcades horizontales constituent des poutrelles ou des ponts qui réunissent les arcades verticales, délimitant ainsi des logettes de forme quadrangulaire.

- Ces logettes (loculi) sont occupées par des divisions de la zone médullaire; elles ont une largeur égale à celle de la zone intermédiaire.

- Les branches dorsale et ventrale de l'artère rénale suivent les arcades dans leurs irradiations et leurs anastomoses.

Les veines se réunissent pour former un systeme veineux dorsal et ventral; la branche dorsale prend une position verticale, en avant de l'uretère, pour rejoindre la branche ventrale et former avec elle la veine rénale.

\section{LES URETÈRES ET LA VESSIE}

L'uretère. - Rien à retoucher aux observations de Lesbre, quant à la situation et aux rapports des uretères. Toutefois, l'uretère gauche est plus court que l'uretère droit. Chez des animaux adultes, l'uretère droit mesure 50 à 55 centimetres de long; l'uretère gauche, 35 à 40 centimètres, dans les deux sexes. Chaque uretère offre un diamètre de 4 millimètres environ.

Ia ressie. - Dans les deux sexes, la vessie est petite, tant dans ses dimensions propres que relatives à la taille de l'animal.

A l'état de contraction, elle est ovale, avec son pôle antérieur légèrement pointu et n'excède jamais le volume d'un ceuf d'oie.

Elle mesure 5 à 7 centimètres de longueur et 4 à 5 centimètres de largeur maxima.

Toutes proportions gardées, elle a une paroi mince, 1 à 1,3 centimètre environ.

Sa position est strictement pelvienne; ce n'est que dans les cas de distension extrême qu'elle fait légèrement saillie hors du pelvis.

Les ligaments latéraux de la vessie se rattachent aux parois latérales plus bas que chez le cheval. Quand elle est distendue, la vessie a une capacité de 600 à 700 centimètres cubes.

Ses rapports avec les autres organes pelviens sont les mêmes que dans les autres espèces domestiques, sauf en haut, où elle contracte des rapports, uniquement avec le rectum, les canaux déférents et la prostate. Les vésicules séminales et l'utricule prostatique font défaut.

La muqueuse vésicale est rosée et devient plus pâle vers le col.

Chez la femelle, la vessie est en rapport, en haut, avec le vagin et, ce n'est que lorsqu'elle est distendue qu'elle atteint le col.

L'urètre chez la femelle. - L'urètre de la femelle a 2 centimètres de diamètre et de 10 à 11 centimètres de longueur environ. L'orifice postérieur de l'urètre s'ouvre sur le plancher du vagin, mesure un centimètre de diamètre et n'est pas dilatable. Il existe un sinus urétral sur le plancher de l'orifice.

\section{GLANDES SURRÉNATES}

Ce sont deux glandes aplaties, de couleur jaune orange ou jaune sale. Elles diffèrent par leur forme et par leur position.

La surrénale droite forme un croissant allongé.

Sa face supérieure est aplatie et se trouve en rapport avec le pilier droit du diaphragme.

La face inférieure est convexe et en rapport avec le duodénum.

Le bord interne est en contact avec la veine cave en arrière, mais sa partie antérieure, ainsi que le pôle antérieur sont incrustés dans la fosse rénale du foie.

Le bord externe est concave et se moule sur la partie antéro-supérieure de la petite courbure du rein droit.

La surrénale gauche est aplatie, discoide, plus longue d'avant en arrière que d'un côté à l'autre. Elle est en rapport, en haut, avec le pilier gauche du diaphragme et se trouve environ à $7-8$ centimètres en avant du pôle antérieur du rein gauche.

Le bord interne est circonscrit par l'angle formé par la veine cave et la veine rénale gauche. En bas, la surrénale gauche est en rapport avec la face supérieure du rumen.

La surrénale droite pèse 40 à 50 grammes et mesure 7 à $8 \times 3$ à 5 centimètres; la surrénale gauche pèse 35 à 40 grammes et mesure 4 à $5 \times 3$ à 4 centimètres.

\section{VASCULERISATON ET INAERVATION}

L'uretère reçoit des branches des artères rénale. spermatique interne; son innervation appartient aux plexus coeliaque et pelvien.

La vessie reçoit des branches de l'artere honteuse interne; son innervation est assurée par le plexus pelvien et par les troisième et quatrième nerfs sacrés.

La surrénale droite reçoit ses vaisseaux de l'aorte; elle ne reçoit pas, comme chez le cheval, de branche de l'artère rénale droite. La surrénale gauche 
reçoit aussi son sang de l'aorte, mais, chez quelques, sujets, I'artère rénale gauche participe à sa vascularisation.

\section{ÉTUDE HISTOLOGIQUE}

L'étude de coupes, après inclusion à la paraffine, a montré les mêmes structures que chez les autres animaux.

\section{BIBLIOGRAPHIE}

CHAUVEAU (A.). - The comparative anatomy of the domes. ticated amimals. 2nd edition. J. and A. Churchill. London, 1891

LESBRE (M -F. X) - Recherches anatomiques sur les Camé. lidés, Archives du Muséum d'Histoire Naturelle, Lyon, 1906, p 106-108.

LEESE ( $\mathbb{A}$-S.) . . $\mathbb{A}$ treatise on the one.humped camel, Haynes and sons, Maiden Lane; Stamford, Lincolnshire, 1927.

ABUL-AZIZ-NEUMANI BEY. - The comparative osteology of . the camel, Cairo 1911 . 